\title{
Intrinsic defects in density waves
}

\author{
S. BRAZOVSKII and N. KIROVA
}

Landau Institute for Theoretical Physics, Moscow, Russia

\begin{abstract}
We present new results on properties of topological defects in Density Wave crystals. For an isolated point - (a $2 \pi$ - soliton) and for a line - (a dislocation) defects the Coulomb interaction dominates in determining distributions of the phase $\varphi$, the potential $\Phi$ and of the accompanying electronic structure. The last shows itself e.g. in a collapse of the density wave gap over a large number of chains around a dislocation. In spin density wave conventional dislocations loose their priority in favor of special topological objects: half - integer dislocations combined with a semi - vortex of a staggered magnetization vector.
\end{abstract}

\section{A single dislocation in charge density wave.}

Properties of dislocations in density wave crystals at low $T$ are determined by the Coulomb forces $[1,2]$ and by screening facilities of free carriers expressed via their normal density $\rho_{n}=1-\rho_{s}$ related to the inverse screening length as $\lambda=\sqrt{\rho_{z}} \kappa, \kappa=\omega_{p} / v_{F}$ (see [3] for precise definitions of all parameters). The dislocation strongly perturbs a region with characteristic lengths $L_{\|}$and $L_{\perp}$ related at low $T$ as $L_{\|}=L_{\perp}^{2} \kappa>L_{\perp}$ which gives a shape tremendously elongated in the (\|) chain axis direction $x$. The $L_{\perp}$ is given either by $\lambda^{-1}$ or by $\xi_{0}=\hbar v_{F} / \Delta_{0}$, both spread over a very large number of chains, $\sim \rho_{n}^{-1 / 2}$ or $\sim \omega_{p} / \Delta_{0}$. The electronic potential $V$ at $x=0, y=R$ (for a dislocation line along $z$-direction) plays a role of the dislocation energy per chain i.e. of it's chemical potential $\mu_{D}$ with respect to aggregated electrons. It decreases at growing $R$ only bejond the screening length $L_{\perp}=R>\lambda^{-1}$. Following [1,2] and new calculations at $R<\xi_{0}$ we have

$$
\begin{gathered}
\lambda R>1: \quad 2 V(R)=\mu_{D}(R)=\frac{\partial W(R)}{\partial\left(\pi R^{2} / s\right)} \sim \frac{T_{c}}{\sqrt{\rho_{n}}} \frac{\xi_{0}}{R} \\
\xi_{0}<R<\lambda^{-1}: \quad V \approx V_{0} \sim \frac{\omega_{p} T_{c}}{\Delta_{0}} ; \quad r<\xi_{0}: V \sim v_{0} \frac{r}{\xi_{0}}
\end{gathered}
$$

Here $T_{c}$ is a $3 \mathrm{~d}$ transition temperature and $s=a_{x} a_{y}$ is the area per chain. The scale $V_{0} \leq \omega_{p}$ is large in compare to $T, T_{c}$ and even to $\Delta_{0}$. Tracing $V(R)$ from very large $R$ we come first to $R=R_{T}$ when $V \sim T$. At $R<R_{T}$ the screening is strongly nonlinear like at commensurability walls [4], Eq. (1) is not applied well and presently we can only say that one sign of carriers will be almost depopulated providing a gate like in semiconductors. This effect may be responsible for a resistivity hysteresis and for the noise in narrow switching samples [5]. At lower $R$ we come to $R \sim R_{c} \sim \xi_{0} / \sqrt{\rho_{n}}$ where $V \sim T_{c}$, or to $R \sim R_{0}$, where $V \sim \Delta_{0}$. In both cases the density wave state is destroyed either by breaking the interchain order for systems with $T_{c}<<T_{M F}$ via 
reaching the critical concentration of solitons (their activation energy is $E_{s} \sim T_{c}$ ) or by breaking the electronic vacuum if $T$ is low or if $V \sim \Delta_{0}$ is reached first in essentially $3 d$ systems. Certainly no pinning can withstand strong tensions $\sim V v_{F} / s$ within these regions.

The low $T$ problem has been solved unexpectedly in a complete way. We find that the dislocation acquires long wings in $y$ direction where amplitude deformations are involved in the form of so called "chordus solitons" [6] for the complex order parameter

$$
\Delta(x, y) / \Delta_{0}=i \cos \varphi_{0}+\sin \varphi_{0} \tanh (x / l), \quad l=\xi_{0} / \sin \varphi_{0}
$$

We have found that

$$
\varphi_{0}=\varphi_{0}(y)=\frac{\pi}{2} \exp \frac{-|y|}{\xi} ; \quad \xi \sim \frac{\xi_{0} T_{c}}{\Delta_{0}}, \quad E_{0}=\Delta_{0} \cos \varphi_{0}
$$

The chordus trajectory (2) provides a "subway connection" between phases $\pm\left[\pi / 2-\varphi_{0}(y)\right]$ and $\pm\left[\pi / 2+\varphi_{0}(y)\right]$ while the intervals $\left[0, \pm\left(\pi / 2-\varphi_{0}\right)\right]$ at $-\infty<x<-l$ and $\left[ \pm\left(\pi-\varphi_{0}\right), \pm \pi\right]$ at $l<x<\infty$ are passed "over the ground" along the circle $|\Delta|=\Delta_{0}$ at $x \rightarrow \pm \infty$ as for a normal dislocation. The phase deficiency $2 \varphi_{0}(y)$ along the line $x=0$ disappears at large $y$.

Every chordus is associated with an intergap state at the energy $E_{0}=E_{0}(y)$ (3). The gap levels are empty at one wing and doubly filled at another one and there is an exactly midgap state $E_{0}=0$ at $y=0$. So the total number of states splited off into the gap per chain along dislocation line is $\xi / a_{y}>>1$ which may be suggested to test optically. Apparently the staircase of levels (3) towards the vacuum $E \rightarrow 0$ at the dislocation line $y \rightarrow 0$ provides an ideal path for a high crossection trapping and for converting free electrons to maintain a dislocation advancing.

\section{Half-integer dislocations/vortices in spin density wave.}

The Coulomb enhancement $\sim \rho_{n}^{-1 / 2}$ of the dislocation energy (1) plays a principal role in spin density wave to bring to life a special combined topological object. This is the half-integer dislocation accompanied by the $180^{\circ}$ rotation of the staggered magnetization $\vec{m}$ [7]. Indeed, the spin density wave order parameter $\vec{\Delta}=\vec{m} \exp i \varphi$ allows for the following three types of self mapping:

i. normal dislocation: $\varphi \rightarrow \varphi+2 \pi, \vec{m}=$ const, $\vec{\Delta} \rightarrow \vec{\Delta}$;

ii. normal $\vec{m}$ - vortex: $\vec{m} \rightarrow \mathcal{O}_{2 \pi} \vec{m}, \varphi=$ const;

iii. combined object : $\varphi \rightarrow \varphi+\pi, \vec{m} \rightarrow \mathcal{O}_{\pi} \vec{m}=-\vec{m}$.

Here $\mathcal{O}_{\theta}$ is the operator of rotation at the angle $\theta$. The combined soliton - the nucleous dislocation loop of type iii. resembles the soliton-twiston complexes suggested for polyacethylene crystals [8]. In case of a spin anisotropy $\vec{m}$ changes the sign along some easy axis and over a definite scale $x_{0}$ of a $180^{\circ}$ domain wall. Then a weak confinement potential will bind ob jects iii. in pairs.

Not far below $T_{M F}$ at $\rho_{n} \sim 1$ the energies of all objects are of the same order. At small $T$ the energy of object $i$. is not affected because no Coulomb force is involved. For objects ii. and iii. most of energy $\sim \rho_{n}^{-1 / 2}$ is associated with the $\varphi$ - counterpart and the energy of $\vec{m}$-rotation may be neglected. At given $R$ the energy of dislocation depends on it's power as $\sim N^{2}$, where the winding number $N=1 / 2$ and $N=1$ for $\pi$ and $2 \pi$ dislocations. As a function of the accumulated number of electrons $Q$ we find

$$
\mu_{D} \sim \partial\left(N^{2} \ln R\right) / \partial(N R) \sim N^{2} / Q, Q \sim N R
$$

and

$$
\mu_{d} \sim \partial\left(N^{2} R \ln R\right) / \partial\left(N R^{2}\right) \sim N^{3 / 2} / Q^{1 / 2}
$$


correspondingly for a dislocation line at a distance $R$ from a surface and for a dislocation ring. In both cases the lowest energy per electron is given by an object with smallest $N=1 / 2$ i.e. by the combined semivortex.

In this way we suggest a natural and rather unambiguous interpretation for a discussed 2fold difference of the narrow band noise frequency versus current ratios for spin density wave in compare to charge density wave. The current conversion or the depinning should proceed via the combined objects iii. We believe that the NMR or AFM - resonance methods will provide a way to see this unusual process. Analogeous combined topological structures have been already observed in $H e^{3}$ phases [9].

Acknowledgements. The authors acknowledge the hospitality of the Laue-Langevin Institute. One of the authors (S.B.) acknowledges the CNRS support during his stay in France where this work was performed.

\section{References}

[1] Brazovskii S., Matveenko S., J. Phys. I, France 1 (1991) 1173.

[2] Brazovskii S., Matveenko S., J. Phys. I, France 2 (1992) 725.

[3] Brazovskii S., These Proceedings.

[4] Artemenko S., Volkov A., Kruglov A., Sov. Phys. JETP 64 (1986) 906.

[5] Pokrovskii V.Ya., Zaitsev-Zotov S.V., Monceau P., Nad' F., These Proceedings.

[6] Brazovskii S., Sov. Phys. JETP 51 (1980) 342

[7] Dsyaloshinskii I.E., JETP Lett. 25 (1977) 98.

[8] Brazovskii S., Kirova N., Synth. Met. 43 (1991) 3639.

[9] Korhonen J.S., Kondo Y., Krusius M., Thuneberg E.V., Volovik G.E., Phys. Rev. $B 47$ (1993) 8868. 